\title{
Ischemic Modified Albumin as A Biomarker in Predicting Oxidative Stress in Patients With Acne Vulgaris
}

\author{
A.A.Ebrahim $^{1}$, A.I.Mustafa ${ }^{1}$, W.A.Abdel-Halim ${ }^{2}$ and D.M.Abo-Ghanema ${ }^{1}$ \\ Dermatology, Venereology and Andrology ,Clinical and Chemical Pathology Dept., Faculty of Medicine, Benha \\ Univ., Benha, Egypt \\ E-Mail: D.M66@gmail.com
}

\begin{abstract}
Acne vulgaris is a cutaneous chronic inflammatory disorder with complex pathogenesis. The aim of this study was to evaluate the serum level of IMA in patients with AV to evaluate its role in AV pathogenesis and its possible relation with oxidative stress markers in the disease. This study was conducted as a case-control study and was conducted on 30 patients suffering from AV (Group A) and 18 apparently healthy individuals of matched age and sex as a control group (Group B). All studied subjects were tested for Serum level of Ischemia modified albumin (IMA), Serum level of Glutathione-STransferase (GST), Serum level of Catalase (CAT) and Serum level of Malondialdehyde (MDA). Serum IMA showed significant positive correlation with GAGS, GSH, MDA and significant negative correlation with CAT. AV grades showed significant positive correlation with GSH, MDA, IMA and significant negative correlation with CAT. No significant correlations were found in IMA with age and duration in AV group Serum IMA then MDA, GSH and CAT might play a role in AV pathogenesis. Moreover, its serum levels could be considered as independent predictor of AV susceptibility, activity and severity.
\end{abstract}

Keywords: Acne vulgaris, oxidative stress, Glutathione-S-Transferase, Catalase.

\section{Introduction}

Acne vulgaris (AV) is a cutaneous chronic inflammatory disorder with complex pathogenesis [1]. It is characterized by the development of seborrhea, comedones, nodules, papules, pustules and cyst. It comes in the areas of the skin with plentiful hair growth such as in the upper chest, back, legs and face [2].

Reactive oxygen species (ROS) are mainly produced by mitochondria, during both physiological and pathological conditions, that is, $\mathrm{O}_{2}{ }^{--}$can be formed by cellular respiration, by lipoxygenases (LOX) and cyclooxygenases (COX) during the arachidonic acid metabolism, and by endothelial and inflammatory cells [3].

The active neutrophils in the inflammatory tissue release ROS. These oxidants attack DNA and/or membrane lipids and cause chemical injury [4]

Ischemia-modified albumin (IMA) is a marker whose levels increase secondary to myocardial and skeletal muscle ischemia. Levels of IMA also increase in diseases involving increased oxidative stress, such as obesity, type 2 diabetes mellitus, hypercholesterolemia, preeclampsia and polycystic ovary syndrome [5]. It may play a role in the pathogenesis of AV [6].

Malondialdehyde (MDA) is the end product of lipid peroxidation and one of the indicators of oxidative stress [7].

Superoxide dismutase-catalase (SOD-CAT) system consists of antioxidant enzymes playing a role in the defence against oxygen toxicity. SOD is an enzyme found in the cytoplasm, and generates hydrogen peroxide, whereas CAT destroys the generated hydrogen peroxide. They have a role in inflammatory diseases [8].

Glutathione (GSH) is the most important hydrophilic antioxidant that protects cells against exogenous and endogenous toxins, including ROS and nitrogen species (RNS) [9]. In addition to its antioxidant activity, GSH has many physiological functions including detoxification of xenobiotics, modulation of redox-regulated signal transduction, regulation of cell proliferation and immune responses [10].

The aim of the present study was to evaluate serum level of IMA and its relation with oxidative stress markers in patients with AV.

\section{Patient and method}

This study was conducted as a case-control study and was conducted on 30 patients suffering from AV (Group A) and 18 apparently healthy individuals of matched age and sex as a control group (Group B). All patients were selected from the outpatient clinic of Dermatology, Venereology and Andrology Department of Benha University Hospitals from January 2020 to February 2020.

The study was approved by the local Ethic Committee of Benha Faculty of Medicine. Informed consent was obtained from each individual before being enrolled in the study.

\section{Inclusion criteria}

- Patients with AV who are diagnosed clinically.

- Patients with different clinical types of AV and with different degrees of severity

\section{Exclusion criteria}

- Subjects with AV on topical treatment in the last two weeks, or systemic treatment in the last month before being enrolled in the study.

- Subjects with history of autoimmune disorders, diabetes mellitus, familial hypercholesterolemia, neoplastic diseases, obesity, liver and kidney diseases.

- Subjects with history of major surgical procedures.

- Pregnant and lactating female patients. 
- Women with history of menstrual irregularities or polycystic ovaries.

\section{All patients were subjected to the followings}

- Full history taking

- Complete general examination: to exclude other systemic diseases as diabetes mellitus, obesity, atherosclerosis, hypertension, rheumatoid arthritis, pregnant and lactating female patients and women with history of menstrual irregularities or polycystic ovaries

- Complete dermatological examination to evaluate the clinical type, site and severity of AV.

- Patients were graded according to GAGS into three groups.

- The total severity score was derived from the summation of six regional subscores. Each was derived by multiplying the factor for each region; (factor for forehead and each cheek was 2, chin and nose was 1 and chest and upper back was 3); by the most heavily weighted lesion within each region (1 for comedones, 2 for papules, 3 for pustules and 4 for nodules). A score of (1-18) is considered mild; (1930) moderate; (31-38) severe; and $>39$ very severe

Laboratory investigations: All studied subjects were tested for:

- Serum level of Ischemia modified albumin (IMA).

- Serum level of Glutathione-S-Transferase (GST).

- Serum level of Catalase (CAT).

- Serum level of Malondialdehyde (MDA).

\section{Results}

Mean disease duration was 4 years; $66.7 \%$ of patients had gradual onset, $33.3 \%$ of patients had sudden onset; $10 \%$ of patients had stationary, $90 \%$ of patients had progressive course; all of them had topical treatment while only one third received systemic treatment (33.3\%). Severity was assessed, $10 \%$ of patients had mild, $53.3 \%$ of patients had moderate and $36.7 \%$ of patients had severe grades. Acne vulgaris had significantly higher frequency of positive family history, smoking, sun exposure and psychological stress when compared to control group ( $\mathrm{P}$ value $<0.05$ each) Acne vulgaris group showed significantly higher GSH, MDA serum levels and significantly lower CAT concentration when compared to control group (P value $0.011,<0.001$ and 0.046 respectively) Acne vulgaris group showed significantly higher IMA when compared to control group ( $\mathrm{P}$ value 0.002 )

Receiver operating characteristic curve of oxidative stress markers and IMA was conducted for discrimination between AV patients and control groups. Serum CAT showed poor AUC (AUC $=0.673$ ). At cut off value of $12.9 \mathrm{ng} / \mathrm{ml}$, sensitivity was $60 \%$, specificity was $66.7 \%$, PPV was $75 \%$, NPV was $50 \%$, accuracy was $62.5 \%$. Serum GSH showed fair AUC (AUC=0.721). At cut off value of $518 \mathrm{ng} / \mathrm{ml}$, sensitivity was $53.3 \%$, specificity was $83.3 \%$, PPV was $84.2 \%$, NPV was $51.7 \%$, accuracy was $64.6 \%$.Serum MDA showed good AUC (AUC=0.881). At cut off value of $49.1 \mathrm{ng} / \mathrm{ml}$, sensitivity was $80 \%$, specificity was $77.8 \%$, PPV was $85.7 \%$, NPV was $70 \%$, accuracy was $79.2 \%$.

Serum IMA showed excellent AUC (AUC=0.962). At cut off value of $68.4 \mathrm{ng} / \mathrm{ml}$, sensitivity was $93.3 \%$, specificity was $77.8 \%$, PPV was $87.5 \%$, NPV was $87.4 \%$, accuracy was $87.5 \%$. The best marker was IMA, then MDA, then GSH and lastly CAT for discrimination between $\mathrm{AV}$ and control groups.Higher IMA concentration was significantly associated with increased acne severity $(\mathrm{p}=0.020)$. Otherwise, no significant differences were found in IMA concentration between studied parameters in AV group ( $p$ value $>0.05$ each ) Serum IMA showed significant positive correlation with GAGS, GSH, MDA and significant negative correlation with CAT. No significant correlations were found in IMA with age of studied patients or disease duration in studied acne patients.

Acne vulgaris grades showed significant positive correlation with GSH, MDA, IMA and significant negative correlation with CAT. No significant correlations were found in IMA with age and duration in AV group.Logistic regression analysis was conducted for prediction of AV development, using age, gender, smoking, FH, sun exposure, stress, GSH, MDA, CAT and IMA as covariates. Positive FH, smoking, sun exposure, excess stress, higher GSH, MDA, IMA were significantly associated with risk of AV occurrence in univariable analysis. However, taking significant risk factors into multivariable analysis revealed that positive $\mathrm{FH}$, smoking, higher MDA and IMA were considered as independent predictors for AV susceptibility.

Ordinal regression analysis was conducted for prediction of Acne severity, using age, gender, smoking, FH, sun exposure, stress, onset, course, duration, GSH, MDA, CAT and IMA as covariates. Lower CAT, higher GSH, MDA and IMA concentrations were associated with more severe AV in uni- and multivariable analyses.

Table (1) Regression analysis for prediction of AV susceptibility.

\begin{tabular}{|c|c|c|c|c|c|c|c|c|}
\hline \multirow{3}{*}{$\begin{array}{l}\text { Variables } \\
\text { Age }\end{array}$} & \multicolumn{4}{|c|}{ Univariable } & \multicolumn{4}{|c|}{ Multivariable } \\
\hline & \multirow{2}{*}{\begin{tabular}{|l|}
$\mathbf{P}$ \\
0.141
\end{tabular}} & \multirow{2}{*}{\begin{tabular}{|c|} 
OR \\
0.878
\end{tabular}} & \multicolumn{2}{|c|}{$95 \% \mathrm{CI}$} & \multirow[t]{2}{*}{$\mathbf{P}$} & \multirow[t]{2}{*}{ OR } & \multicolumn{2}{|c|}{$95 \% \mathrm{CI}$} \\
\hline & & & 0.739 & 1.044 & & & & \\
\hline Gender & 0.256 & 2.000 & 0.605 & 6.612 & & & & \\
\hline $\begin{array}{l}\text { Positive family } \\
\text { history }\end{array}$ & 0.048 & 3.455 & 1.010 & 11.813 & 0.008 & 1.190 & 1.047 & 1.354 \\
\hline Smoking & 0.030 & 2.118 & 1.189 & 3.467 & 0.014 & 1.192 & 1.036 & 1.372 \\
\hline
\end{tabular}


Table (1) Continue

\begin{tabular}{lcccccccc}
\hline Excess sun exposure & 0.042 & 2.500 & 1.985 & 3.331 & 0.126 & 1.191 & 0.521 & 1.389 \\
Excess stress & 0.033 & 2.701 & 1.082 & 6.747 & 0.285 & 1.079 & 0.938 & 1.241 \\
GSH-ST & 0.015 & 1.009 & 1.002 & 1.017 & 0.255 & 0.999 & 0.998 & 1.022 \\
MDA & 0.001 & 1.128 & 1.052 & 1.210 & 0.001 & 1.011 & 1.005 & 1.017 \\
CAT & 0.139 & 0.874 & 0.731 & 1.045 & & & & \\
IMA & 0.004 & 1.109 & 1.034 & 1.190 & 0.037 & 1.116 & 1.064 & 1.281 \\
\hline
\end{tabular}

OR, odds ratio; CI, confidence interval.

Table (2) Regression analysis for prediction of severity of AV.

\begin{tabular}{|c|c|c|c|c|c|c|c|c|}
\hline \multirow{3}{*}{$\begin{array}{l}\text { Variables } \\
\text { Age }\end{array}$} & \multicolumn{4}{|c|}{ Univariable } & \multicolumn{4}{|c|}{ Multivariable } \\
\hline & \multirow{2}{*}{$\begin{array}{c}\mathbf{P} \\
0.106\end{array}$} & \multirow{2}{*}{$\begin{array}{c}\text { OR } \\
1.110\end{array}$} & \multicolumn{2}{|c|}{$95 \% \mathrm{CI}$} & \multirow[t]{2}{*}{$\mathbf{P}$} & \multirow[t]{2}{*}{$\mathbf{O R}$} & \multicolumn{2}{|c|}{$95 \% \mathrm{CI}$} \\
\hline & & & 0.978 & 1.259 & & & & \\
\hline Gender & 0.205 & 1.698 & 0.580 & 3.967 & & & & \\
\hline Positive family history & 0.565 & 1.281 & 0.551 & 2.976 & & & & \\
\hline Smoking & 0.789 & 0.894 & 0.392 & 2.037 & & & & \\
\hline Excess sun exposure & 0.836 & 1.096 & 0.460 & 2.608 & & & & \\
\hline Excess stress & 0.282 & 1.594 & 0.682 & 3.724 & & & & \\
\hline Onset & 0.836 & 1.096 & 0.460 & 2.608 & & & & \\
\hline Course & 0.442 & 1.696 & 0.441 & 6.524 & & & & \\
\hline Duration & 0.308 & 1.091 & 0.923 & 1.289 & & & & \\
\hline GSH-ST & 0.008 & 1.006 & 1.002 & 1.011 & 0.012 & 1.012 & 1.003 & 1.034 \\
\hline MDA & 0.006 & 1.042 & 1.012 & 1.073 & 0.039 & 1.051 & 1.012 & 1.070 \\
\hline CAT & 0.015 & 0.850 & 0.746 & 0.968 & 0.039 & 0.542 & 0.190 & 0.924 \\
\hline IMA & 0.022 & 1.006 & 1.001 & 1.012 & 0.039 & 1.035 & 1.024 & 1.086 \\
\hline
\end{tabular}

OR, odds ratio; CI, confidence interval.

\section{Discussion}

The oxygen radical scavenging activity of GSH directly facilitates ROS neutralization and the repair of ROS-induced damage [11]. In addition to its antioxidant activity, GSH has many physiological functions including detoxification of xenobiotics, modulation of redox-regulated signal transduction, regulation of cell proliferation and immune responses [10].

Ischemia-modified albumin is assumed "N-terminal modified" albumin which is generated immediately following myocardial ischemia [12]. Levels of IMA also increase in diseases involving increased oxidative stress, such as obesity, type 2 diabetes mellitus, hypercholesterolemia, preeclampsia and polycystic ovary syndrome [13]. IMA, an oxidative stress parameter, may play a role in the pathogenesis of AV [6]. It is a new biochemical marker. It is associated with inflammation [14].

In the present study we aimed at assessment of serum of IMA in patients with AV to evaluate its role in AV pathogenesis and its possible relation with oxidative stress markers in the disease.

Oxygen free radicals damage cellular functions depending on time and duration of exposure. Oxidative stress is a term used to indicate the imbalance between concentration of oxygen free radicals and concentration of antioxidative defence mechanisms in the body. This imbalance plays a role in various pathologies [15].

In oxidative stress, the balance between antioxidant defense mechanism and free radical formation is disturbed and endothelial dysfunction occurs [16]. Acne patients not only have local tissue damage but are also under systemic oxidative stress [17]. Oxidative stress is important in the progression of acne as well as the etiopathogenesis. The skin is exposed to oxidative damage of ROS, which is produced by endogenous and exogenous reasons [18].

Oxidative stress has a role of in the pathogenesis of rheumatoid arthritis (RH) and osteoarthritis (OA), and that lipid peroxidation (LPO) markers and antioxidants can serve as surrogate markers for disease activity [19].

In the present study, we found that AV group showed significantly higher GSH, MDA and significantly lower CAT concentration when compared to control group.

In line with our work, Arican et al. [4] measured oxidative stress parameters such as CAT and MDA in patients with AV. They reported that while levels of CAT statistically decreased significantly, levels of MDA increased statistically, indicating oxidative stress in acne patients.

Also, Sarici et al. [20] noted that the serum levels of MDA activity in the patients with AV were significantly higher than those of the controls. A significantly lower CAT activity was found in the patient group than in the control group.

Sahib et al. [21] reported significantly higher serum MDA levels but GSH levels were lower than in healthy controls.

In contrast to our results, Başak et al. [22] found higher CAT activity in leucocytes of patients with acne. 
Many inflammatory, metabolic and infectious diseases affect the skin and joints. Most of these, such as rheumatoid arthritis and systemic lupus erythaematosus are considered to be rheumatic conditions with secondary skin involvement. However, several primary cutaneous diseases are associated with arthritis and may even present with joint symptoms prior to cutaneous lesions. Common skin disorders, such as acne and psoriasis, have well-known musculoskeletal manifestations [23].

There is a well described association of arthritis with severe skin inflammation and acne [24]. Concequently, $\mathrm{AV}$ has a relation with osteoarthritis (OA) and rheumatic arthritis (RA) due to the similarity in their pathogenesis through oxidative stress. As in arthritis, the increased oxidative stress in RA and OA patients led to compensatory changes in the levels of antioxidants. These findings confirm the role of oxidative stress in the pathogenesis of RA and OA, and that lipid peroxidation (LPO) markers and antioxidants can serve as surrogate markers for disease activity.

El-barbary et al. [19] demonstrated significantly high serum levels of MDA and in both OA and RA groups as compared with the healthy control group. There was insignificant difference in MDA serum levels between the RA and OA groups. They found significantly lower erythrocyte SOD, CAT activities and GSH level in OA and RA groups compared to the control group.

Akyol et al. [25] found a remarkable elevation in MDA levels in patients with RA compared to controls. The increased ROS levels in RA may result in a prooxidation environment, which in turn could result in increased MDA levels. As a result, LPO may have a role in the pathogenesis of the RA [19]. The increased MDA level in OA patients coincides with the results of Rubyk et al. [26] who reported significantly increased serum MDA levels in OA patients compared to controls. Thus, these findings are in keeping with possible evidence of free radical production and damage in OA.

In the current study, AV group showed significantly higher IMA when compared to control group. Higher IMA concentration was significantly associated with higher AV grades.

In agreement of our study, Akyurek et al. [27] stated that IMA associated with ischemia and oxidative stress may play an important role in the etiopathogenesis of AV. It also suggests that measuring the plasma levels of these markers may be useful in assessing the severity and progression of the disease. The authors reported that IMA levels were significantly higher in patients than controls and its level also related to the disease severity.

Also, Gürel et al. [6] found the mean IMA level in patients with AV was significantly higher than in the control group. This indicates that IMA, an oxidative stress parameter, may play a role in the pathogenesis of AV.

High IMA levels have been also determined in various inflammatory dermatological diseases such as psoriasis, alopecia areata, vitiligo and Behçet's disease [6].
Ozdemir et al. [28] reported that IMA levels increase in patients with psoriasis as an adaptive response to oxidative stress and systemic inflammation.

In the current work, AV grades showed significant positive correlation with GSH, MDA, IMA and significant negative correlation with CAT.

Our results came in agreement with Al-Shobaili et al. [29] regarding MDA correlation with AV grades as the authors illustrated that degree of lipid peroxidation in the progress of AV was analyzed by lipid peroxidative end products, MDA. MDA levels were significantly increased with the increase of the disease activity. MDA level was significantly high in AV patients when compared with normal human subjects.

Al-Shobaili et al. [29] regarding GSH correlation with AV grades, found levels of serum GSH were significantly reduced in mild or severe acne patients compared with normal subjects of the same age group. They demonstrated that mild acne patients showed insignificant decreased in the serum GSH when compared with normal human subjects. The decreased serum activities of GSH levels suggest that the antioxidant balance is compromised in acne that may lead to increased ROS levels and, thus, contribute to increased oxidative stress. In addition, these findings also indicate that oxidative stress is increased in $\mathrm{AV}$ and is associated with increased disease activity.

Regarding the present study, IMA levels were considered as independent predicators for AV susceptibility and severity.

The present result was in agreement of Gürel et al. [6] who determined no significant differences in IMA levels among the AV subgroups. This suggests that the effect of IMA levels in patients with AV may be independent of the severity of the disease, as well as indicating the possible involvement of compensatory mechanisms in the body.

\section{Conclussion}

From the results of present study, it is concluded that: Serum IMA then MDA, GSH and CAT might play a role in AV pathogenesis. Moreover, its serum levels could be considered as independent predictor of AV susceptibility, activity and severity.

\section{Recommendations}

The results of the current study should be interpreted in light of its limitations, as the present study included a relatively small sample size.

Further studies are needed to evaluate tissue expression of IMA, MDA, GSH and CAT in vitro in active and inactive AV.

Further studies are needed to investigate the precise mechanisms by which IMA, MDA, GSH and CAT contribute to the pathogenesis of AV.

Further studies should be done to asses the role of IMA, MDA, GSH blockers in treatment of AV.

Further studies should be done to measure IMA, MDA, GSH and CAT before and after systemic treatment for acne. 


\section{References}

[1] T. Cong, D. Hao, X. Wen, From pathogenesis of acne vulgaris to anti-acne agents. Arch Dermatol Res;VOL. 311(5), PP.337-349,2019.

[2] B. Ravisankar, H .Liu, R .Shinde, The amino acid sensor GCN2 inhibits inflammatory responses to apoptotic cells promoting tolerance and suppressing systemic autoimmunity. PNAS, Vol. 112(34),PP.10774-10779,2015.

[3] K. Al-Gubory, C. Garrel, P .Faure, Roles of antioxidant enzymes in corpus luteum rescue from reactive oxygen species-induced oxidative stress. Reprod Biomed online, Vol. 25, PP.551-560,2012.

[4] O .Arican, E. Kurutas, S .Sasmaz, Oxidative stress in patients with acne vulgaris. Mediators Inflamm, Vol. 2005,PP.380-384,2005.

[5] G .Caglar, E. Oztas, D .Karadag, Ischemia-modified albumin and cardiovascular risk markers in polycystic ovary syndrome with or without insulin resistance. Fertil steril, Vol. 95,PP.310-313,2011.

[6] G .Gürel, M. Karadöl, C .Bal, Evaluation of ischemia-modified albumin levels in acne vulgaris patients. Turkish Arch Dermatol Venerol/Turkderm, Vol. 53,PP.15-24,2019.

[7] H. Kang, D. Kim, B. Lee, Urinary N-acetyl-beta-Dglucosaminidase and malondialdehyde as a markers of renal damage in burned patients. J Korean Med Sci; VOL.16, PP.598,2001.

[8] J. Mates, C .Perez-Gomez , I. De Castro , Antioxidant enzymes and human disease. Clin Bioch ; VOL.32,PP.595-603,1999.

[9] M. Jozefczak, T. Remans, J .Vangronsveld, Glutathione is a key player in metal-induced oxidative stress defenses. Int J Mol Sci, Vol. 13, PP.3145-3175,2012.

[10]F. Santangelo, V .Witko-Sarsat, T. Drueke, Restoring glutathione as a therapeutic strategy in chronic kidney disease. Nephrol Dial Transplant, Vol.19,PP.1951-1955,2004.

[11]A. Pastore, G. Federici, E. Bertini, Analysis of glutathione: implication in redox and detoxification. Clin Chim Acta, Vol. 333, PP.19-39,2003.

[12] O. Ismail , O. Bulent ,Ischemia-Modified Albumin as a Marker of Acute Coronary Syndrome: The Case for Revising the Concept of (N-Terminal Modification) to (Fatty Acid Occupation) of Albumin. Dis Marker, Vol.35, PP.1-9,2017.

[13] S. Piva, M .Duarte, I. Da Cruz, Ischemia-modified albumin as an oxidative stress biomarker in obesity. Clin Biochem; VOL.44, PP.345-347,2011.

[14]Z. Karatas, T .Baysal, F .Şap, Increased ischaemia-modified albumin is associated with inflammation in acute rheumatic fever. Cardiol Young, Vol. 24(3),PP.430-436,2014.
[15]S .Lee , I .Blair , Oxidative DNA damage and cardiovascular disease. Trends Cardiovasc Med, Vol. 11, PP.148-155,2001.

[16]P. Ozuguz, S. Dogruk Kacar, O. Ekiz, Evaluation of serum vitamins $\mathrm{A}$ and $\mathrm{E}$ and zinc levels according to the severity of acne vulgaris. Cutaneous Ocular Toxicol, Vol. 33, PP.99-102,2014.

[17] S .Awad, H .Morsy, A. Sayed, Oxidative stress and psychiatric morbidity in patients with facial acne. J Cosmet Dermatol, Vol. 17(2),PP.203-208,2018.

[18]B. Dreno, What is new in the pathophysiology of acne, an overview. JEADV, Vol. 31(5), PP.812,2017 .

[19]A. El-barbary, M .Abdel- Khalek, A. Elsalawy, , Assessment of lipid peroxidation and antioxidant status in rheumatoid arthritis and osteoarthritis patients. Egy Rheumatol, Vol. 33(4) , PP.179$185,2011$.

[20] G .Sarici, S. Cinar, F .Armutcu, Oxidative stress in acne vulgaris. J Eur Acad Dermatol Venereol, Vol. 24, PP.763-767,2010.

[21]A .Sahib, H. Al-Anbari , A. Raghif , Oxidative stress in acne vulgaris: an important therapeutic target. J Mol Pathophysiol; VOL.2, PP.27-31,2013.

[22]P .Basak, F. Gultekin , I .Kilinc, The role of the antioxidative defense system in papulopustular acne. J Dermatol, Vol. 28, PP.123-127,2001.

[23] J .Levin , F .Werth ,Skin disorders with arthritis. Best Pract Res Clin Rheumatol, Vol. 20(4), PP.809$826,2006$.

[24]C .Bengham , Acne causing joint inflammation? J Dermatol, Vol. 15, PP.20-25,2011.

[25] O .Akyol, N. Iscedilçi, I .Temel, The relationships between plasma and erythrocyte antioxidant enzymes and lipid peroxidation in patients with rheumatoid arthritis. Joint Bone Spine, Vol. 68, PP.311-317,2001.

[26] B. Rubyk, N. Fil'chagin , R. Sabadyshin, Change in lipid peroxidation in patients with primary osteoarthrosis deformans Ter Arkh, Vol. 60 (9), PP.110-113,1988.

[27]F .Akyurek, G. Kurtipek, H. Kurku, Assessment of ADMA, IMA, and Vitamin A and E Levels in Patients with Acne Vulgaris. JCD, Vol.60, PP.16,2020.

[28] M. Özdemir, A. Kiyici, A. Balevi, Assessment of ischaemia-modified albumin level in patients with psoriasis. Clinical and Experimental Dermatology: Clin Dermatol, Vol. 37, PP.610-614,2012.

[29]H. Al-Shobaili, A .Alzolibani, A. Al Robaee, Biochemical Markers of Oxidative and Nitrosative Stress in Acne Vulgaris: Correlation With Disease Activity. JCLA, Vol. 27(1), PP.45-52,2013. 